\title{
Dementia in Swedish Twins: Predicting Incident Cases
}

\author{
Margaret Gatz $\cdot$ Chandra A. Reynolds • \\ Deborah Finkel • Nancy L. Pedersen • \\ Ellen Walters
}

Received: 22 July 2009/Accepted: 8 October 2010/Published online: 24 October 2010

(c) The Author(s) 2010. This article is published with open access at Springerlink.com

\begin{abstract}
Thirty same-sex twin pairs were identified in which both members were assessed at baseline and one twin subsequently developed dementia, at least 3 years subsequent to the baseline measurement, while the partner remained cognitively intact for at least three additional years. Eighteen of the 30 cases were diagnosed with Alzheimer's disease. Baseline assessments, conducted when twins' average age was $70.6(\mathrm{SD}=6.8)$, included a mailed questionnaire and in-person testing. Which twin would develop dementia was predicted by less favorable lipid values (higher apoB, ratio of apoB to apoA1, and total cholesterol), poorer grip strength, and-to a lesser extenthigher emotionality on the EAS Temperament Scale. Given the long preclinical period that characterizes Alzheimer's disease, these findings may suggest late life risk factors for dementia, or may reflect changes that are part of preclinical disease.
\end{abstract}

Edited by George Vogler.

M. Gatz $(\bowtie) \cdot$ N. L. Pedersen · E. Walters

Department of Psychology, University of Southern California,

3620 S. McClintock Avenue, Los Angeles 90089-1061,

CA, USA

e-mail: gatz@usc.edu

M. Gatz · N. L. Pedersen

Department of Medical Epidemiology and Biostatistics,

Karolinska Institutet, Stockholm, Sweden

C. A. Reynolds

Department of Psychology, University of California-Riverside, Riverside, CA, USA

D. Finkel

Department of Psychology, Indiana University Southeast,

New Albany, IN, USA
Keywords Twins - Dementia - Alzheimer's disease · Cholesterol · Grip strength · Emotionality

\section{Introduction}

Dementia is the diagnostic term for a group of disorders entailing progressive decline in memory and other cognitive abilities sufficient to interfere with daily, social, and occupational functioning. Two main types of dementia are Alzheimer's disease (AD) and vascular dementia (VaD). Alzheimer's disease, which accounts for up to two-thirds of all cases of dementia, is thought to result from a pathological cascade initiated by deposition in the brain of a protein called beta-amyloid (Hardy 2006). These deposits are associated with oxidative injury, inflammatory activity, and acetycholine deficits that lead to a loss of neurons and account for the cognitive and behavioral dysfunction that is observed clinically. For a summary of clinical and pathophysiological features of Alzheimer's disease, see Cummings and Cole (2002). The onset of Alzheimer's disease is insidious, with a long preclinical period, and the course is gradual. See Fig. 1.

Vascular dementia is characterized by cerebral infarcts that are evident in neuroimaging. The onset of vascular dementia may be more abrupt and the course more stepwise compared to Alzheimer's disease. Risk factors for vascular dementia include midlife hypertension, diabetes mellitus, and hyperlipidemia. Vascular risk factors have been linked epidemiologically to $\mathrm{AD}$, not only to vascular dementia, and there is increasing evidence that infarction and $\mathrm{AD}$ pathology combine additively to accelerate symptoms of dementia (Chui et al. 2006).

The strongest risk factor for dementia is age. In North America and Europe, 6-10\% of those aged 65 and older are 


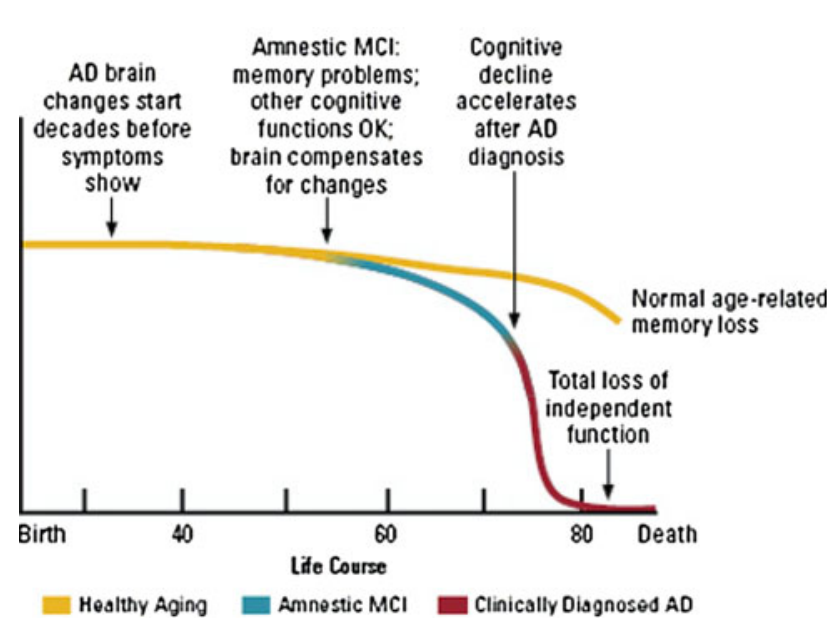

Fig. 1 Change over time from healthy aging to Alzheimer's disease (AD). Some people's course entails gradually moving to mild cognitive impairment and then possibly making the transition to AD. Other people continue on a course of healthy aging, showing only normal age-related changes in cognition. Figure from National Institute on Aging (2008, p. 29)

affected by dementia. The rate is low among those in their sixties but then doubles every 5 years (Ferri et al. 2005). The next strongest risk factor for dementia is genetics. However, approximately only $2 \%$ of $\mathrm{AD}$ cases are caused by specific genetic mutations, and 50\% of AD cases do not carry the apolipoprotein $\varepsilon 4$ polymorphism that is the most wellestablished genetic risk factor for AD (Tanzi and Bertram 2005). Consequently, like other chronic diseases, the cause of dementia almost certainly lies in a complex combination of genetic influences and environmental exposures, and these exposures likely accumulate over the entire lifespan. Teasing apart these factors is a huge challenge, and one with important implications for preventive intervention.

The interest in potentially modifiable risk factors for $\mathrm{AD}$ was given impetus by the observation that level of education was related to risk of $\mathrm{AD}$, such that disease risk was nearly double for those with low education (Katzman 1993). This observation led naturally to studying the effect of other forms of intellectual stimulation such as leisure activities as possible protective factors to lower rates of dementia (Fabrigoule et al. 1995). Vascular risk factors have also received increased attention as risk factors not only for vascular dementia but also for Alzheimer's disease. Given the role of the apolipoprotein $\varepsilon 4$ as a genetic risk factor, research has naturally examined lipid profiles and subsequent Alzheimer's disease (Kivipelto and Solomon 2006). The idea that proneness to psychological distress might serve as a risk factor for Alzheimer's disease, possibly through deleterious effects of stress on the brain, has found support in studies such as Wilson et al. (2002) who reported increased risk of AD onset among those with higher baseline levels of depressive symptoms, with up to 7 years of follow-up.
Study of Dementia in Swedish Twins

Soon after the Swedish Adoption/Twin Study of Aging (SATSA) was launched (Pedersen et al. this issue), we proposed adding an ancillary Study of Dementia in Swedish Twins. The study became known as SALZA. A twin study of dementia permits addressing the following research questions: (a) what is the relative contribution of genetic and environmental influences for Alzheimer's disease and other dementias; (b) what specific risk and protective factors can be identified through comparing demented twins to their non-demented twin partners, thereby controlling for familial influences; (c) longitudinally, what preclinical differences characterize the twin who will develop dementia compared to their partner who remains dementia-free; and (d) how do cognitively intact partners of probands compare to cognitively intact partners of non-demented twins. The rationale for using a twin design to identify risk and protective factors has long been argued, but the demands of implementing such studies has been formidable, including the length of time required to acquire an appropriate sample (see Breitner et al. 1993).

The SATSA data collection timeline is shown in Fig. 2. Beginning in 1987, we telephoned all non-responders to the first two mailed questionnaires, Q1 and Q2, and anyone who had refused to participate in the first two in-person testing sessions, IPT1 or IPT2, and asked whether the individual was willing to take part in a brief telephone interview. If the person was unable, we asked to speak with a relative. The telephone interview included a mental status test focused on memory abilities and a few questions about health and daily activities (Gatz et al. 1995). Relatives were asked whether the twin had difficulties in memory or thinking and whether these difficulties led to problems in daily activities, to moving into a different type of housing, or to visiting a physician to evaluate the cognitive difficulties. For those participating in the SATSA in-person testing, the mental status examination that is part of the IPT protocol was used

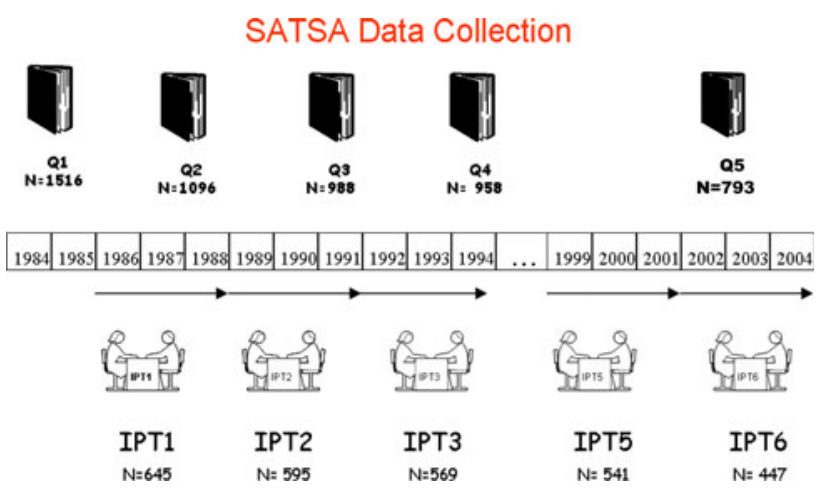

Fig. 2 Data collection timeline for SATSA. Dementia cases were identified at each of these measurement occasions 
as the twin's cognitive screening, in conjunction with the nurse's clinical impressions from the entire IPT session. Anyone whose telephone interview or IPT results suggested onset of cognitive problems sufficient to interfere with daily activities was invited to participate in a complete in-person clinical evaluation. The results of the clinical evaluation were considered at a consensus diagnostic conference, where a clinical diagnosis was assigned.

At each new wave of SATSA, i.e., Q3, IPT3, and Q4, new non-responders were screened and those whose results suggested cognitive problems were referred for a complete clinical evaluation. When IPT4 was discontinued, the telephone screening protocol was used to screen all members of the SATSA IPT sample. Again, anyone whose results suggested cognitive problems was referred for clinical evaluation and diagnosis. After SATSA data collection started up again, at both IPT5 and IPT6 additional cases of dementia were identified, using similar procedures to obtain a consensus clinical diagnosis.

By the end of IPT4, we had identified 75 twins with dementia, half of whom were diagnosed with Alzheimer's disease. The probandwise concordance rate for Alzheimer's disease among monozygotic (MZ) pairs was 67\%; the corresponding figure for dizygotic pairs was $22 \%$. Heritability of liability to Alzheimer's disease was estimated to be 0.74 ; to any dementia, 0.43 , with the other variance attributable to environmental influences (Gatz et al. 1997). Concordance rates do not incorporate information about the time it takes for the unaffected partner to become demented. Thus, for example if pairs were re-classified as discordant when the difference in age at onset between the first and second twin to develop dementia was 7 or more years, then concordance for Alzheimer's disease would drop to $36 \%$ for MZ pairs (Posner et al. 1999). This observation constitutes powerful evidence that there is a role for non-genetic factors in determining risk of developing $\mathrm{AD}$, with those non-genetic risk factors including environmental exposures, epigenetic factors, and chance.

In many of our analyses of risk factors, we included discordant pairs from both SALZA and OCTO-Twin (Johansson this issue). We looked first at low education as a risk factor, where we found that having low education approximately doubled risk of Alzheimer's disease. Although the number of twin pairs who differed in amount of formal education was small, it was still the case that having a lower education than one's twin partner was associated with approximately doubling risk of Alzheimer's disease (Gatz et al. 2001).

Also with a combined SALZA and OCTO-Twin sample, we looked at leisure activities in which twins engaged before age 40, based on self-report data that were collected by the Swedish Twin Registry in 1967. Those who engaged in a greater number of different activities had a significantly lower risk of Alzheimer's disease more than 20 years later. If considered separately, this finding held for women but not men, and was most pronounced for activities that were categorized as "intellectual-cultural" (Crowe et al. 2003).

Recently with a combined SALZA and OCTO-Twin sample, we reported that higher body mass index (BMI) measured in 1963 predicted both later $\mathrm{AD}$ and later $\mathrm{VaD}$ (Hassing et al. 2009).

A strength of these analyses is that the risk factor was assessed decades before dementia onset. Thus, it is unlikely that the dementia caused differences in education, leisure activities, or BMI. A limitation to these analyses is that they were based on a cross-sectional assessment of dementia status. Therefore, there could be biases based on differential survival.

\section{Predicting incident dementia}

There are now sufficient waves of SATSA data to accumulate a sample of incident cases where the individuals participated in at least one wave of SATSA in-person testing, were cognitively intact at that time, and subsequently developed dementia. These cases are the focus of the new results reported in this article.

We test three predictors of incident dementia-serum lipid values, grip strength, and temperament-all measured at the baseline SATSA assessment.

Two reviews of cholesterol and dementia suggest that the evidence for a relationship between cholesterol and incident dementia is ambiguous (Kivipelto and Solomon 2006; Purnell et al. 2009). Some reports find total cholesterol or triglycerides, especially if measured at midlife, to be significant risk factors for $\mathrm{AD}$, while other studies find no relationship, and at least one study finds high cholesterol to be protective.

Mid-life grip strength is a good predictor of later functional limitations and disability (Rantanen et al. 1999). For this reason, we thought it might be a useful measure to include among predictors of dementia onset. We found two prior reports confirming decline in grip strength as a predictor of incident AD (Buchman et al. 2007; Taaffe et al. 2009), while Wang et al. (2006) found that poor grip strength predicted progression from mild cognitive impairment to dementia.

Our third predictor was the emotionality scale from the EAS Temperament Survey (Buss and Plomin 1984), selected to identify those who perceived and approached life as more stressful. Stress is associated with activation of the hypothalamic-pituitary-adrenal (HPA) axis, releasing glucocorticoid hormones. In turn glucocorticoids can cause loss of neurons, particularly in the hippocampus, a key brain structure for memory (Sapolsky 1996). Investigators interested in psychological stress as a risk factor for dementia have most often chosen to study depression, although, as summarized by Jorm (2001), evidence with 
respect to depression as a risk factor for dementia is inconsistent. A measure of depressive symptoms was unavailable prospectively, and we selected Emotionality as the best indicator in the SATSA questionnaires of proneness to experience life as stressful.

Our hypothesis was that those twins who subsequently developed dementia scored less favorably at the baseline assessment of these three predictors than their cognitively intact twin partners. For purposes of comparison, we also included in the incident cases analyses three measures corresponding to findings from the prevalent case analyses-education, engagement in cultural activities, and body mass index.

\section{Method}

\section{Participants}

The SATSA sample includes 2845 individuals from same sex twin pairs who were enrolled in the Swedish Twin Registry in the 1960s and 1970s. Included in SATSA are all pairs from the Swedish Twin Registry in which members of the pair were separated before age 10, and an equal number of pairs reared together matched on year of birth, sex, and county of birth. Longitudinal study of the SATSA sample continues (see Pedersen, this issue), with 861 individuals participating in at least one in-person assessment and 2,211 with data from at least one questionnaire assessment.

A total of 1089 individuals from the SATSA sample have been determined to meet criteria for dementia $(n=215)$ or to be non-demented $(n=874)$ based on screening and diagnostic procedures described above. Non-responders to SATSA questionnaire or in-person data collection are overrepresented among the dementia cases; thus, these numbers should not be used to form a prevalence rate. Of the 215 cases, 139 were prevalent, i.e., no SATSA assessment occurred prior to dementia diagnosis, and 76 were incident, i.e., there was at least one wave of SATSA data collection.

For purposes of the present analyses, from the incident cases, we identified 22 discordant pairs where the case developed dementia after participating in at least one wave of SATSA, and the twin partner also participated in at least one wave of SATSA and was alive and non-demented at the next assessment after the proband's age of onset. We also identified 8 concordant pairs who at one point met that same definition but the twin partner subsequently went on to develop dementia more than 3 years later. This three-year criterion to classify pairs as discordant follows the suggestion of Breitner (Breitner et al. 1994). Demographic characteristics of these 30 pairs are shown in Table 1. Clinical diagnoses included: 18 Alzheimer's disease, 4 vascular dementia, 2 mixed AD and vascular, 2 dementia due to secondary causes, and 4 with dementia type unspecified.

The other incident cases were not suitable for a discordant pairs analysis. Another 26 cases were members of discordant pairs, but in 17 pairs the non-demented twin died before the proband's dementia onset and in 9 pairs the non-demented twin was lost to follow-up for other reasons before the proband's dementia onset. There were 3 concordant pairs where the difference in age of onset was less than 3 years, and there were 6 concordant pairs where one twin's dementia was incident but the other twin's dementia was classified as prevalent.

Table 1 Demographic characteristics of pairs for discordant pairs analysis $(N=30$ pairs $)$

\begin{tabular}{|c|c|c|c|c|}
\hline \multicolumn{5}{|l|}{ Sex } \\
\hline Men & \multicolumn{4}{|c|}{$N=7$ pairs } \\
\hline Women & \multicolumn{4}{|c|}{$N=23$ pairs } \\
\hline \multicolumn{5}{|l|}{ Zygosity } \\
\hline Monozygotic & \multicolumn{4}{|c|}{$N=11$ pairs } \\
\hline Dizygotic & \multicolumn{4}{|c|}{$N=18$ pairs } \\
\hline Indeterminate & \multicolumn{4}{|c|}{$N=1$ pair } \\
\hline & Mean & SD & Minimum & Maximum \\
\hline Proband age & 70.66 & 6.82 & 57 & 88 \\
\hline Partner age & 70.63 & 6.87 & 57 & 88 \\
\hline Age of onset for dementia in proband & 77.02 & 6.71 & 63 & 90 \\
\hline Difference in age of onset in concordant pairs & 5.19 & 3.32 & 3 & 13 \\
\hline $\begin{array}{l}\text { Difference between age of onset in proband and last } \\
\text { measurement in partner in discordant pairs }\end{array}$ & 5.94 & 3.27 & 1.72 & 14.44 \\
\hline
\end{tabular}

Notes: The proband is the demented twin in discordant pairs $(N=22)$ or the first twin to become demented in concordant pairs $(N=8)$. Partner is the non-demented twin in discordant pairs or the second twin to become demented in concordant pairs. Age refers to the age at which the predictor variable was assessed; for probands, this occurred during the preclinical phase 


\section{Measures}

Lipid levels were determined from serum samples extracted from whole blood taken at SATSA in-person testing sessions and frozen at $-70^{\circ} \mathrm{C}$. Standard procedures were used to establish lipid levels (Iliadou et al. 2001), including serum triglycerides, total cholesterol, high density lipoprotein (HDL) and apolipoproteins A1 and B (apoA1 and apoB). All lipid values reported are in $\mathrm{mmol} / \mathrm{L}$ units with the exception of apoA 1 and apoB which are reported in $\mathrm{g} / \mathrm{L}$ units. Prior to analyses, log-transformations were applied to triglycerides, HDL, apoB, and the ratio of apoB to apoA1 values to reduce non-normality.

Grip strength was measured during SATSA in-person testing using a dynamometer. Participants were seated and used a table as support for the elbow. The maximum of six scores (three from each hand) was selected (Finkel et al. 2003)

Emotionality was measured with four items: "often get worried," "often feel dissatisfied," "worried by everyday events," and "am easily upset." These items are from the EAS Temperament Survey (Buss and Pomin 1984), which measures Emotionality, Activity, and Sociability. Each item is answered on a five-point likert scale indicating how characteristic of him- or herself the respondent believes the item to be, the responses are summed, and the total is divided by four. The EAS was included on the mailed SATSA questionnaire.

Education was measured as years of formal education.

Engagement in cultural activities was measured with five items from the Moos Family Environment Scale (Moos and Moos 1981) phrased to reflect current activities. The five items encompassed discussing social and political issues, attending lectures or theater, having intellectual discussions, interest in culture, and interest in the arts. These questions were included on the mailed SATSA questionnaire.
BMI was calculated from height and weight using the formula WTKG/(HTCM/100) ${ }^{2}$, where WTKG is weight in kilograms and HTCM is height in centimeters.

\section{Analyses}

The question of interest was whether the twins who subsequently would develop dementia differed from their partners on the predictor variables. To test the research question, we carried out discordant pairs analyses, comparing scores for the preclinical twin and the partner, and testing the significance of within-pair differences using a matched $t$-test. $95 \%$ confidence intervals are provided, and Cohen's d was calculated as a measure of effect size. Pairs were excluded from analysis if the onset of dementia was less than 3 years after the predictor variable was measured. This criterion reduced to 23 the number of pairs for predictors measured at in-person testing. However, the addition of this criterion did not substantially alter the results. Follow up analyses controlled for sex and for the duration between time of measurement of the predictor and age of onset of dementia. As results were not changed, analyses without covariates are presented here.

\section{Results}

Results are shown in Table 2. The preclinical twin had a significantly less favorable cholesterol profile on apoB, ratio of apoB to apoA1, and total cholesterol. Effect sizes $=0.63,0.64$, and 0.50 , respectively. Intra-pair differences on HDL and apoA1 were not significant.

On grip strength, of 23 pairs, 22 were discordant for grip strength at the in-person testing session, prior to age of onset of dementia in the proband. On average, grip strength was significantly higher in unaffected members (see Table 2).

Table 2 Results from discordant pairs analyses

\begin{tabular}{|c|c|c|c|c|}
\hline Predictor variable & $N$ pairs & Mean intrapair difference & $95 \%$ confidence interval & Matched $t$-test \\
\hline Years of education & 28 & 0.07 & $-0.66,0.80$ & $0.20, p=0.5785$ \\
\hline Engagement in cultural activities & 20 & 0.50 & $-0.51,1.51$ & $1.00, p=0.8351$ \\
\hline BMI & 29 & 0.29 & $-1.52,2.11$ & $0.33, p=0.6281$ \\
\hline HDL & 20 & -0.03 & $-0.12,0.07$ & $-0.62, p=0.2713$ \\
\hline ApoA1 & 23 & -0.02 & $-0.09,0.06$ & $-0.46, p=0.3250$ \\
\hline ApoB & 23 & -0.09 & $-0.15,-0.03$ & $-3.02, p=0.0062$ \\
\hline Ratio B/A1 & 23 & -0.08 & $-0.13,-0.02$ & $-3.05, p=0.0059$ \\
\hline Total cholesterol & 23 & -0.56 & $-1.04,-0.07$ & $-2.40, p=0.0253$ \\
\hline Grip strength & 23 & 3.35 & $0.60,6.09$ & $2.53, p=0.0190$ \\
\hline Emotionality & 28 & -0.36 & $-0.72,0.006$ & $-2.02, p=0.0536$ \\
\hline
\end{tabular}

Notes: A positive difference indicates that the partner scored higher than the preclinical twin; while a negative difference indicates that the preclinical twin scored higher than the partner. BMI body mass index, $H D L$ high density lipoprotein, $A p o A 1$ apolipoprotein A1, ApoB apolipoprotein B 
Effect size $=0.53$. In 8 pairs, the preclinical twin had higher grip strength; in 14 pairs, the partner had higher grip strength.

On EAS Temperament, of 28 pairs, 25 had discordant scores on the baseline questionnaire. In 15 pairs, the preclinical twin had higher emotionality; in 10 pairs, the partner had higher emotionality. While at a $p$ of 0.0536 , the significance of the matched t-test is marginal (see Table 2), the effect size is 0.38 .

Notably, in this incident discordant pairs design, neither years of education, or engagement in cultural activities or BMI measured at SATSA baseline significantly distinguished between the twin who was subsequently to develop dementia and the unaffected partner (see Table 2).

\section{Discussion}

Taken together, our past findings plus new results from SATSA twins in the Study of Dementia in Swedish Twins begin to give a picture of how the etiology of dementia might be understood. First, genetic risk plays a marked role, particularly for Alzheimer's disease. Second, nongenetic or environmental factors are influential, possibly as a trigger for the timing of dementia onset. Third, nongenetic factors must be considered in relation to the life course. In conjunction with Fig. 1, which illustrates cognitive function over the life course, different influences on cognition will be important at different ages along the horizontal axis. Thus, as suggested by the results reported here, there may be different sets of predictor variables for prevalent as compared to incident cases.

Much prior research, including our own, has established exposures during midlife that constitute risks for dementia, while finding that the same exposures later in life may not be significant risks or may even reverse direction. For example, our own group (Hassing et al. 2009) and others (e.g., Whitmer et al. 2005) have found overweight in middle age to increase risk of dementia. On the other hand, in the Religious Order Study, drop in BMI predicted incident dementia during an average of five and a half years of follow-up (Buchman et al. 2005). Buchman and colleagues reasoned that the loss of body mass may reflect preclinical processes in which factors that contribute to development of AD also lead to loss of body mass. A similar pattern of results may pertain for blood pressure, where midlife hypertension receives some support as a risk factor for Alzheimer's disease (e.g., Kivipelto et al. 2001), yet preclinical cases have been found to have low blood pressure 6 years prior to dementia onset (Qiu et al. 2003).

In the Study of Dementia in Swedish Twins, among the midlife risk factors for dementia are low education (Gatz et al. 2001), low engagement in leisure activities- especially intellectual and cultural activities (Crowe et al. 2003), and high BMI in midlife (Hassing et al. 2009). In the present analyses, education, engagement in cultural activities, and BMI measured on average five and a half years prior to dementia onset did not prove significant predictors of incident dementia.

For incident cases, in the present analyses, predictors of which twins would develop dementia included a more unfavorable lipid profile, poorer grip strength, and higher emotionality. For the lipid values and grip strength findings, effect sizes were medium to large; for emotionality, small. Some but not all lipid values proved predictive. Neither HDL nor apoA1 were significantly protective; however, apoB, the ratio of apoB to apoA1, and total cholesterol were significant predictors of which twin would become demented. This result is provocative in suggesting that elevated "bad" cholesterol continues to be a risk factor even late in life, although level of "good" cholesterol does not appear to be important. Past evidence summarized by Purnell et al. (2009) about the relationship between lipid levels and incident dementia has been highly inconclusive; thus, our results clearly bear replication and extension before suggesting preventive interventions. Recently, our group completed a more extensive analysis of lipids and cognitive change (Reynolds et al. 2010). Although the main focus was the relationship of lipid profiles to normative cognitive aging, the results included an analysis of pairs discordant for incident dementia. Unlike the present study, these analyses excluded pairs where one twin developed dementia and the twin partner subsequently developed dementia more than 3 years later. The findings were similar for total cholesterol and apoB, although not for the ratio of apoB to apoA1.

Consistent with Buchman et al. (2007), and Taaffe et al. (2009), we found poor grip strength to be a significant predictor of incident AD. Given the long preclinical period that characterizes Alzheimer's disease, for our incident cases it is not possible to distinguish between true risk factors and changes that are part of dementia onset. Especially since Wang et al. (2006) found grip strength particularly predictive of dementia among those with mild cognitive impairment, it is possible that grip strength represents a marker during the preclinical course of dementia rather than a change that occurs prior to the processes associated with the development of dementia.

Emotionality predicted incident dementia, albeit at $p=0.0536$, with Cohen's $\mathrm{d}=0.38$ and power $=0.336$. Similarly, Wilson et al. (2002) reported increased risk of AD onset with higher levels of depressive symptoms. Based on the fact that number of depressive symptoms did not continue to increase across a four-year period before the onset of Alzheimer's disease, Wilson et al. (2008) subsequently argued the depression was a true risk factor 
and not an early manifestation of the dementia. At the same time, it is also plausible that individuals who are beginning to sense changes in their own cognitive function would react with worry and distress. Thus, we would conclude that it is ambiguous in our results whether emotionality constitutes a true risk factor or represents an early marker of the disease that is beginning to develop.

Limitations of these findings include the small number of pairs. At the same time, the criteria for drawing the matched pairs were rigorous, and we are confident that the pairs qualify as discordant for dementia onset. The matched twin design has the advantage of controlling for familial influences. At the same time, since twins tend to be similar on many of the risk factors, the design may actually overcontrol for familiality. For these reasons, the findings are provocative and suggest paths for further research on the timing of risk factors and distinguishing risk factors from preclinical changes. We continue to add discordant pairs as SATSA twins are followed longitudinally. Larger samples would permit, for example, looking at pairs by zygosity, examining environmental factors that might modify genetic influences, and looking separately at types of dementia. Finally, these results underline the complexity in identifying modifiable risk factors for dementia on which recommendations for health behavior can be based. In particular, taken together with our previous reports, the results imply that different interventions might be directed toward midlife and toward late life.

Acknowledgments The Study of Dementia in Swedish Twins has been supported by NIH Grants No. AG08724 and AG031262, and by a Zenith award from the Alzheimer's Association ZEN-02-3895. The Swedish Adoption/Twin Study of Aging (SATSA) has been supported by NIH Grants No. AG04563 and AG10175, Swedish Social Sciences Research Council, MacArthur Foundation Research Network on Successful Aging, and the Swedish Research Council. Cholesterol analyses were supported by NIH Grant No. AG028555. We wish also to acknowledge the important intellectual contribution to this work from Professor Stig Berg, who died in March, 2009.

Open Access This article is distributed under the terms of the Creative Commons Attribution Noncommercial License which permits any noncommercial use, distribution, and reproduction in any medium, provided the original author(s) and source are credited.

\section{References}

Breitner JCS, Gatz M, Bergem ALM, Christian JC, Mortimer JA, McClearn GE, Heston LL, Welsh KA, Anthony JC, Folstein MF, Radebaugh TS (1993) The use of twin cohorts for research in Alzheimer's disease. Neurology 43:261-267

Breitner JCS, Gau BA, Welsh KA, Plassman BL, McDonald WM, Helms MJ, Anthony JC (1994) Inverse association of antiinflammatory treatments and Alzheimer's disease: initial results of a co-twin control study. Neurology 44:227-232

Buchman AS, Wilson RS, Bienias JL, Shah RC, Evans DA, Bennett DA (2005) Change in body mass index and risk of incident Alzheimer disease. Neurology 65:892-897
Buchman AS, Wilson RS, Boyle PA, Bienias JL, Bennett DA (2007) Grip strength and the risk of incident Alzheimer's disease. Neuroepidemiology 29:66-73

Buss AH, Plomin R (1984) Temperament: early developing personality traits. Erlbaum, Mahwah, NJ

Chui HC, Zarow C, Mack WJ, Ellis WG, Zheng L, Jagust WJ et al (2006) Cognitive impact of subcortical vascular and Alzheimer disease pathology. Ann Neurol 60:677-687

Crowe M, Andel R, Pedersen NL, Johansson B, Gatz M (2003) Does participation in leisure activities lead to reduced risk of Alzheimer's disease? A prospective study of Swedish twins. J Gerontol B Psychol Sci Soc Sci 58:P249-P255

Cummings JL, Cole G (2002) Alzheimer disease. JAMA 287: 2335-2338

Fabrigoule C, Letenneur L, Dartigues JF, Zarrouk M, Commenges D, Barberger-Gateau P (1995) Social and leisure activities and risk of dementia: a prospective longitudinal study. J Am Geriatr Soc 43:485-490

Ferri CP, Prince M, Brayne C, Brodaty H, Fratiglioni L, Ganguli M et al (2005) Global prevalence of dementia: a Delphi consensus study. Lancet 366:2112-2117

Finkel D, Pedersen NL, Reynolds CA, Berg S, de Faire U, Svartengren M (2003) Genetic and environmental influences on decline in biobehavioral markers of aging. Behav Genet 33:107-123

Gatz M, Reynolds C, Nikolic J, Lowe B, Karel M, Pedersen N (1995) An empirical test of telephone screening to identify potential dementia cases. Int Psychogeriatr 7:429-437

Gatz M, Pedersen NL, Berg S, Johansson B, Johansson K, Mortimer JA, Posner SF, Viitanen M, Winblad B, Ahlbom A (1997) Heritability for Alzheimer's disease: the Study of Dementia in Swedish Twins. J Gerontol A Biol Sci Med Sci 52:M117-M125

Gatz M, Svedberg P, Pedersen NL, Mortimer JA, Berg S, Johansson B (2001) Education and the risk of Alzheimer's disease: findings from the Study of Dementia in Swedish Twins. J Gerontol B Psychol Sci Soc Sci 56:P292-P300

Hardy J (2006) Has the amyloid cascade hypothesis for Alzheimer's disease been proved? Curr Alzheimer Res 3:71-73

Hassing LB, Dahl AK, Thorvaldsson V, Berg S, Gatz M, Pedersen NL, Johansson B (2009) Overweight in midlife and risk of dementia: A 40-year follow-up study. Int J Obesity. Advance online publication, 9 June 2009. doi:10.1038/ijo.2009.104

Iliadou A, Lichtenstein P, de Faire U, Pedersen NL (2001) Variation in genetic and environmental influences in serum lipid and apolipoprotein levels across the lifespan in Swedish male and female twins. Am J Med Genet 102:48-58

Jorm AF (2001) History of depression as a risk factor for dementia: an updated review. Aust N Z J Psychiatry 35:776-781

Katzman R (1993) Education and the prevalence of dementia and Alzheimer's disease. Neurology 43:13-20

Kivipelto M, Solomon A (2006) Cholesterol as a risk factor for Alzheimer's disease-epidemiological evidence. Acta Neurolologica Scandinavia 114(Suppl. 185):50-57

Kivipelto M, Helkala E-L, Laakso MP, Hänninen T, Hallikainen M, Alhainen K, Soininen H, Tuomilehto J, Nissien A (2001) Midlife vascular risk factors and Alzheimer's disease in later life: longitudinal, population based study. Br Med J 322:1447-1451

Moos RH, Moos BS (1981) Manual for the family environment scale. Consulting Psychologists Press, Palo Alto, CA

National Institute on Aging, Alzheimer's Disease Education and Referral Center. (2008, September). Alzheimer's disease: Unraveling the mystery. U.S. Department of Health and Human Services NIH Publication Number: 08-3782

Posner SF, Pedersen NL, Gatz M (1999) The application of life table analysis to the onset of dementia in a genetically informative design. Am J Med Genet (Neuropsychiatric Genetics) 88: 207-210 
Purnell C, Gao S, Callahan CM, Hendrie HC (2009) Cardiovascular risk factors and incident Alzheimer disease: a systematic review of the literature. Alzheimer Dis Assoc Disord 23:1-10

Qiu C, von Strauss E, Fastbom J, Winblad B, Fratiglioni L (2003) Low blood pressure and risk of dementia in the Kungsholmen Project: a 6-Year follow-up study. Arch Neurol 60:223-228

Rantanen T, Guralnik JM, Foley D, Masaki K, Leveille S, Curb JD, White L (1999) Midlife hand grip strength as a predictor of old age disability. JAMA 281:558-560

Reynolds CA, Gatz M, Prince JA, Berg S, Pedersen NL (2010) Serum lipid levels and cognitive change in late life. J Am Geriatr Soc 58:501-509

Sapolsky RM (1996) Why stress is bad for your brain. Science 273:749-750

Taaffe D, Irie F, Masakl K, Abbott R, Petrovitch H, Ross W, White L (2009) Handgrip strength and future dementia in elderly men: the Honolulu-Asia Ageing Study. J Sci Med Sport 12(1):S3
Tanzi RE, Bertram L (2005) Twenty years of the Alzheimer's disease amyloid hypothesis: a genetic perspective. Cell 120:545-555

Wang L, Larson EB, Bowen JD, van Belle G (2006) Performancebased physical function and future dementia in older people. Arch Intern Med 166:1115-1120

Whitmer RA, Gunderson EP, Barrett-Connor E, Quesenberry CP Jr, Yaffe K (2005) Obesity in middle age and future risk of dementia: a 27 year longitudinal population based study. Br Med J 330:1360-1362

Wilson RS, Barnes LL, Mendes de Leon CF, Aggarwal NT, Schneider JS, Bach J, Pilat J, Beckett LA, Arnold SE, Evans DA, Bennett DA (2002) Depressive symptoms, cognitive decline, and risk of AD in older persons. Neurology 59:364-370

Wilson RS, Arnold SE, Beck TL, Bienias JL, Bennett DA (2008) Change in depressive symptoms during the prodromal phase of Alzheimer's disease. Arch Gen Psychiatry 65:439-446 